\title{
Daylighting System Based on DSP and FPGA of New Intelligent Building
}

\author{
Wang Jingqiu \\ School of Automation \\ Beijing Institute of Technology \\ Beijing, China \\ xxlylf@sina.com
}

\author{
Li Xuan, Zhang Yunqi \\ School of Optoelectronics, School of Software \\ Beijing Institute of Technology \\ Beijing, China \\ li_xuan1989@hotmail.com
}

\begin{abstract}
The daylighting system of new intelligent building applies an automatic control system based on DSP and FPGA. It adjusts optical paths accordingly with the angle direction of various optical system arrays, collecting as much solar energy as possible and transferring it efficiently to each room in need, in order to achieve a healthy, hygienic, energy-saving, and environmentally-friendly light source. While lighting is not needed, it can transfer the solar energy into other kinds of useful energy to store with installed equipment such as photocells, solar water heaters etc. In the meantime, the system can also make automatic adjustments for overall light intensity within the room under user's settings according to surrounding light intensity.
\end{abstract}

Keywords- Intelligent building, daylighting, DSP, FPGA

\section{INTRODUCTION}

The daylighting system of new intelligent building adopts DSP and FPGA as the operation and control centre. DSP system collects information for real-time hardware computing. The results are transmitted to FPGA for the adjustment of the control mechanisms. The terminal sensor module is used to control the light intensity in the room by judging that of the user's surroundings. The terminal sensor makes use of a silicon photocell to collect the data of light intensity around; the central sensor employs a servo motor to change the angle of the photocell and meanwhile deliver the message to DSP system.

The number of motors is quite large, and the length of time required by the control signal is very demanding, accurate to $0.5 \mu \mathrm{s}$. Thus if we only choose DSP, multiple motors control cannot be realized, because of the limitation of the quantity of pins. What is more, the system structural principle restricts the time length of the control signal to not less than $5 \mu$ s. However, FPGA integrated circuits can supply adequate pins to control hundreds of motors. In addition, the time length can be controlled accurately to $0.01 \mu \mathrm{s}$. In this case, FPGA is fully able to meet the demand of the system, and increase the overall stability.

\section{System Design}

\section{A. Optical Design Module}

In Figure 1, 1 is a sphere concave mirror. 2 and 2' are both plane mirrors. 3 and 3' are the roof. Bold solid lines are the edge rays reflected by mirror 2 . Bold dotted lines are the edge rays reflected by mirror 2'. Thin dotted lines are the normal of different incident rays to the concave mirror.

2 and 2' are installed on different levels of the roof but in the same plane. The plane mirrors collect sunlight irradiated on the roof as well as the sensors. Meanwhile, the sensors transmit the sunlight signals to the servos, which are attached to the mirrors and then drive them to rotating around a certain angle. Thus, reflected by the mirrors and then by the concave mirror, the rays go into a given hole on the roof as the light source of the building [1,2].

Figure 1 is used to show that different installation heights lead to different illuminated area on the roof. Sunlight is regarded to be parallel; so are the reflected rays of the mirror. In Figure 1, the shadows represent the illuminated area on the roof after the bold dotted and the bold solid rays reflected by the concave mirror respectively. By comparing illuminated area on the roof, we can find the best installation height $[3,4]$.

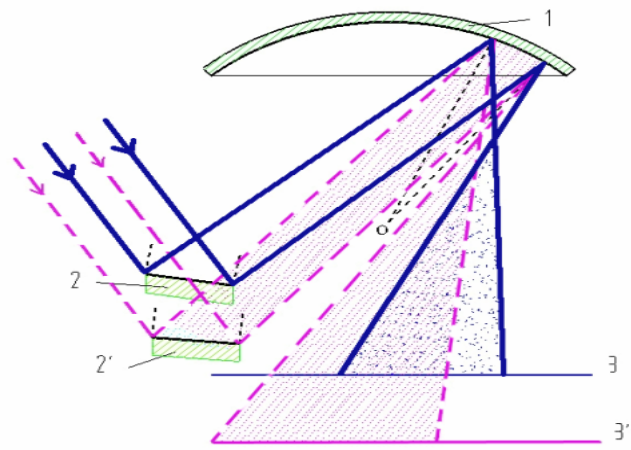

Figure 1. Analog light path

In Figure 2, $\vec{M}$ is the shaft of Motor 1, whose direction is the same as the y-axis in the coordinate system. Motor 1 is joined to the sensor. $\vec{N}$ is the shaft of Motor 2, whose direction is the same as the $\mathrm{z}$-axis in the coordinate system. $\vec{A}$ is the original normal of the sensor, whose direction is the same as the x-axis in the coordinate system. $\vec{M}$ maintains perpendicular to $\vec{A}$ all the time. $\alpha$ is the angle Motor 1 rotates. $\beta$ is the angle Motor 2 rotates.

After receiving the signals from the sensor, the motor rotates. Since it is attached to the circuit board of the sensor, the rotation changes the normal direction of the sensor. 
Assuming Motor 1 rotates $\alpha$ around the shaft, thus $\vec{A}$ rotates to $\vec{A}_{1}$; Motor 2 rotates $\beta$ around the shaft, thus $\vec{A}_{1}$ rotates to $\vec{A}_{2} \cdot \vec{A}_{2}$ is the direction of sunlight rays.

In Figure $2, \vec{A}, \vec{A}_{1}$ and $\vec{A}_{2}$ are all unit vector.

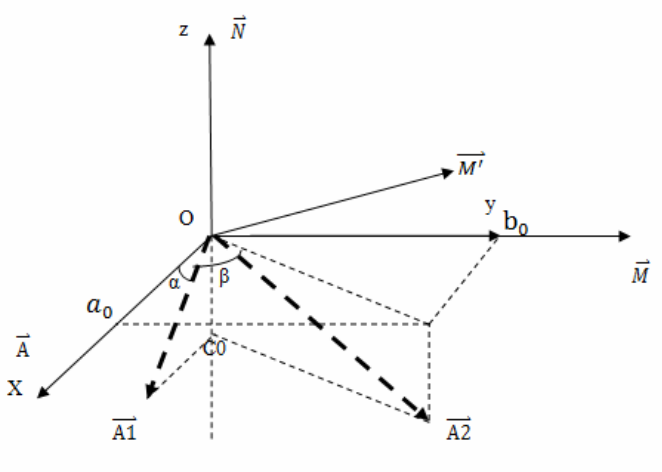

Figure 2. Vector calculation of the servo

Now we can calculate the direction vector of sunlight $\vec{A}_{2}$, which is a unit vector.

In Figure 3, 1 is a concave mirror with a radius of $R .2$ is a plane mirror. We build a coordinate system with the centre of the plane mirror $O$ as its origin. The height from the roof and the mirror is $h$, and the distance between the mirror and the centre of the hole is $L . O_{1}$ is the focal point of the concave mirror, which is $\mathrm{R} / 2$ away from its centre. If the reflected rays from the centre of the plane mirror pass $O_{1}$, they can incident into the hole vertically. The height from the roof to $O_{1}$ is $H$.

The sunlight incident vector can be measured by the sensor, and its unit vector is .

The reflected vector is shown in Figure 3, that is, $(0, L$, $\left.H-h+\frac{R}{2}\right)$. Then we can calculate its unit vector, setting it as $\vec{B}\left(0, b_{1}, c_{1}\right)$.

The normal vector is what we seek, set as $\vec{C}(x, y, z)$. Because the incident ray, the reflected ray, and the normal are in the same plane, we can obtain:

We can find the best position of the plane mirror by calculating the final normal vector $\vec{C}(x, y, z)$. Next we have the rotation angle of the plane mirror.

In Figure 4, $\vec{C}(x, y, z)$ is the final normal vector of the plane mirror, and $\mathrm{x}, \mathrm{y}$, and $\mathrm{z}$ are calculated specific values. $\vec{M}$ is the shaft of Motor 1, whose direction is the same as the $y$-axis in the coordinate system. Motor 1 is joined to the mirror. $\vec{N}$ is the shaft of Motor 2, whose direction is the same as the z-axis in the coordinate system. $\vec{D}$ is the original normal of the mirror, whose direction is the same as the $\mathrm{x}$-axis in the coordinate system. $\vec{D}$ maintains perpendicular to $\vec{M}$ all the time.

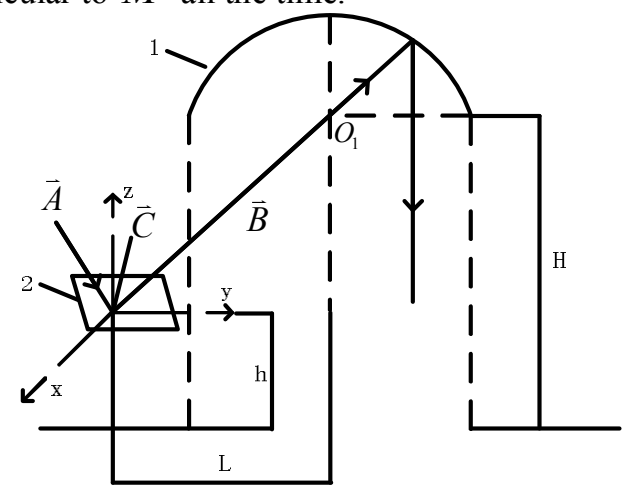

Figure 3. Vector calculation of angle control

First $\vec{D}$ rotates $\theta$ around $\vec{M}$ and then $\gamma$ around $\vec{N}$. Substituting $x, y$, and $z$, we have:

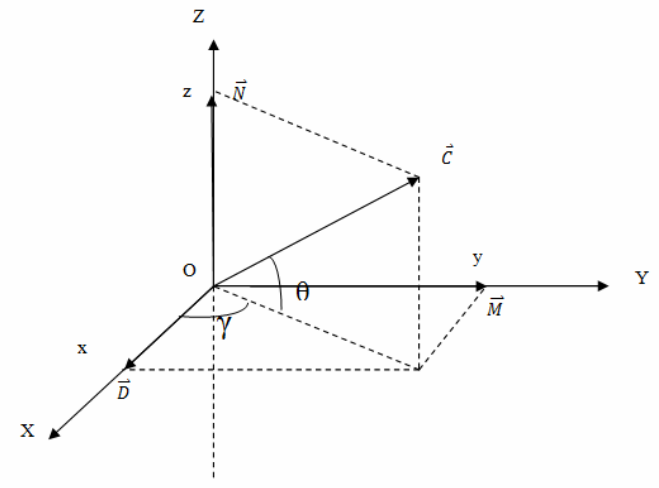

Figure 4. Vector relations of the plane mirror and the motors

\section{B. Mechanical Building Blocks}

This device is used to lead sunlight into the optical channel in the building. The position of the sun changes continuously in the daytime. Therefore, the plane mirror mechanism must adjust the angle with the sun's movement, which requires two or more degrees of freedom. This device has two degrees of freedom. In other words, the mirror mechanism contains two servos, adjusting $x$ direction and $y$ direction respectively. Under this condition, however the sun moves, sunlight rays can be reflected to the concave mirror and then converged into the channel.

\section{Sensor Module}

Sensor 1, Sensor_2, and Sensor_ 3 are three light sensor chips (OPT 101$)$. Changes in external light intensity alter the sensor output voltage. From the Figure 5 below, we can draw the following conclusions. $U 1$ and $u 2$, the output of Sensor_1 and Sensor_2 respectively, are the input of LM324N (A). The corresponding output is $u 1-u 2$ (In this figure, they are amplified 10 times. However they are only doubled, if the values of $R 2, R 4, R 6$, and $R 8$ are all $1 \mathrm{~K} \Omega$ ). Similarly, the input of LM324N (D) is the output voltages of Sensor_1 and 
Sensor 3, and its output is $u 1-u 3$. LM324 (B) and LM358 (A) are voltage followers, whose input is the output of LM324N (A) and LM324N (D) respectively. There are capacitors linking their output to the ground, filtering. The circuit composed by $R 16$ and $D 1$ is used to exploit the fixed voltage $0.7 \mathrm{~V}$ across the diode as the threshold in the next comparison circuit. The function of LM324N (C) and LM358 (B) is to compare the voltage after followers and filter with $0.7 \mathrm{~V}$ [5].

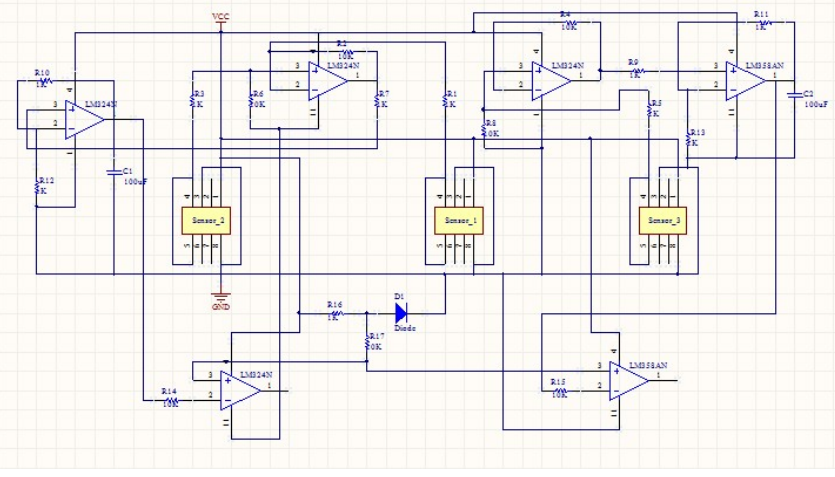

Figure 5. Sensor circuit design

In Figure 6, Channel $A$ and Channel $B$ are sensor waveforms under different light conditions, mixed with power frequency noise and waveform distortion. Channel $C$ presents the result of $A-B$, and the dotted line represents $0.7 \mathrm{~V}$. Obviously, the result of logic operation should be square wave pulse of power frequency, but not be low-level signal (which means the light density measured by Sensor_1 is weaker than that measured by Sensor_2).

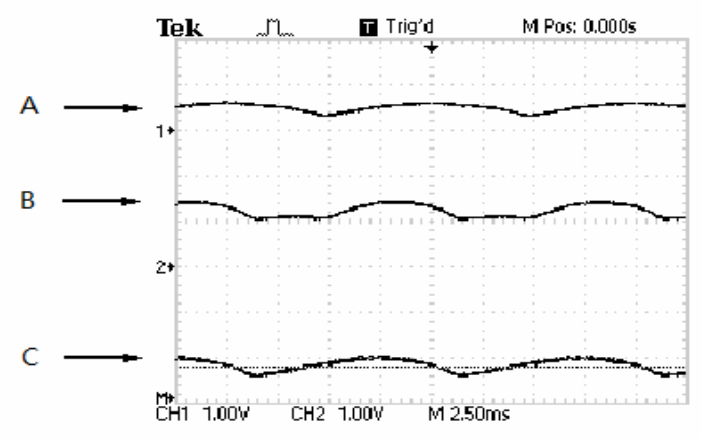

Figure 6. Scope test results

With the limitations of mechanical structure, the volume of the circuits in the sensor part needs reducing. So we obtain the sensor output voltages directly by using $\mathrm{A} / \mathrm{D}$ conversion, and reduce power frequency noise and waveform distortion by using RC filter. The picture below shows parts of the principle.

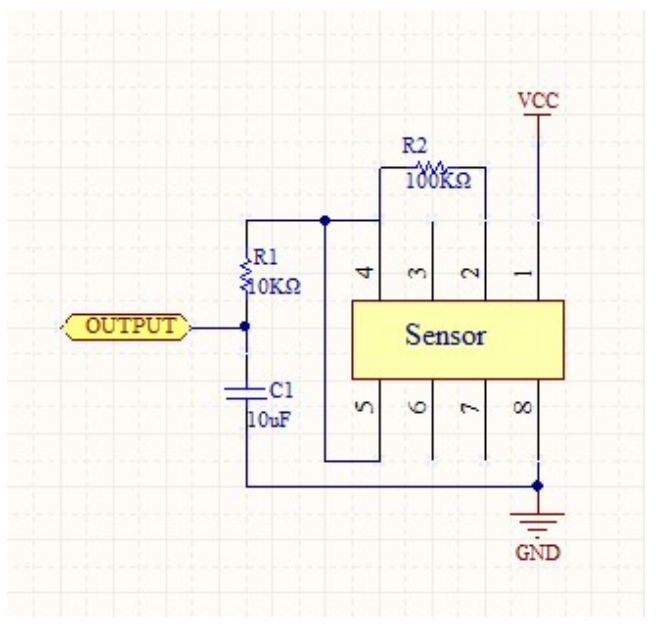

Figure 7. The schematic picture of sensor circuits

In Figure 8, Channel $B$ tests the original output signal of the sensor. Clearly, the amplitude of noise is about $1 / 7$ of the signal average. Channel $A$ measures the filtered signal. There are few fluctuations in the signal. So A/D conversion can be done. It proves that the filtered signal does not tremble and the value does not beat during the conversion in 12 bit precision.

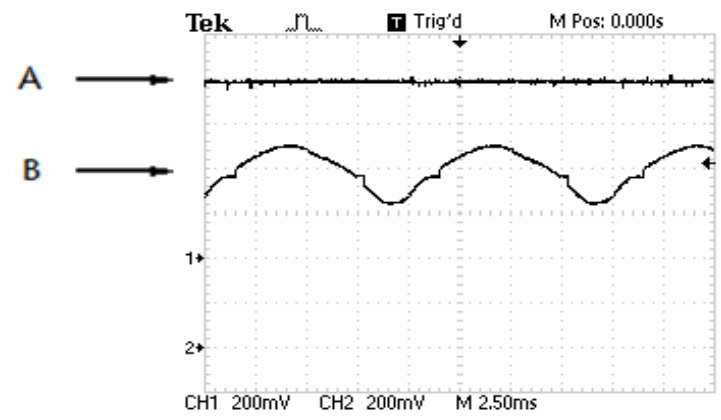

Figure 8. Test results after filtering

\section{DSP Data Processing Module (56F8013)}

DSP is a suitable microprocessor for the digital signal processing operations. It can implement lots of digital signal processing algorithms timely and rapidly. 56F8013 can sample in three channels at the rate of $1 \mathrm{KHz}$. The built-in hardware multiplier allows the algorithm to run fast. When the motor rotates, DSP calculates at the same time. It can update the stored value by comparing it with the value of the sensor received signal. Then it transmits the angle signal, corresponding to the maximum of light density, to FPGA.

\section{E. FPGA Control Module (EP2C5Q208C8N)}

FPGA is a kind of semicustom circuits in the field of Application Specific Integrated Circuits (ASIC). It overcomes not only the disadvantages of custom circuits, but also the shortage of gate circuits in the original programmable devices. The selected chip EP2C5Q208C8N 
has adequate $\mathrm{I} / \mathrm{O}$ pins as well as build-in FLASH memories. Thus the development cycle can be largely shortened and the costs are greatly reduced. In the daylighting system of new intelligent building, FPGA is mainly used to generate multiple signals for motor group control in the light of the DSP operation results.

As the executive terminal, FPGA converts the difference signal into motor control signal and export PWM wave to control the steering of the sensors and the optical instruments.

\section{SCHEME COMPARISON}

\section{A. The Choice of the Top Reflection Mirror}

At first the convex mirror was chosen as a reflection mirror on the top, while the plane mirror could not be too large during the measurement if it required normal incidence, or it would cause scattering. Thus, the concave mirror was taken into account for it cannot only gather light but also get more rays incident vertically.

\section{B. The Choice of Curvature of the Top Reflection Mirror}

A concave mirror with a larger curvature would be a better choice, even though it costs more than that with a smaller one. It deserves for the reason that the former one can introduce more light to the optical channel.

\section{The Argument of the Auxiliary Effect of the Optical Fiber}

Optical fiber was once designed in the system to help rays incident vertically at the beginning, and in that case the optical path could be altered freely. Although the improvement of accuracy would make sense, the high cost should be taken into account, as well as that the reflection angle was the same as the incident angle through the optical fiber. In conclusion, the optical fiber has not been added into the system.

\section{The Argument of Three or Two Degrees of Freedom}

Originally, we considered to apply multi-stage servos with three degrees of freedom to control the plane mirrors. However, the practice proves that the plane mirror with two degrees of freedom could satisfy the requirements of the system quite well. To simplify the mechanical structure, we choose the servos with two degrees of freedom to control.

\section{E. The Choice of the Stepper Motor}

The stepper motor provides more accurate angle output than the common servo does. However, at the experimental stage, its large volume and the complexity in control are not suitable for the model's mechanical fabrication, for which the common servo would be a better choice temporarily. In the stage of practical application, the stepper motor is supposed to be a powerful improvement.

\section{TECHNOLOGY AND INNOVATION}

\section{A. An Innovative Method to Improve the Lighting Condition for Buildings}

The sunlight is introduced to the channel after reflected as the light source. Not only does it reduce the consumption of energy, but also benefits to people physically and mentally. In addition, the full usage of natural light could prevent people from the pollution of discomfort glare $[6,7]$.

\section{B. The Use of PI Control and Hysteresis Loop Comparison}

Due to the delay of the servo itself, there might be shocks during the practice. To make sure that the sensors and the plane mirrors could rotate an accurate angle, PI control is added into the system. To prevent the servo rotation angle from small interference, we bring in hysteresis loop comparison to keep the servos working steadily in the range of error.

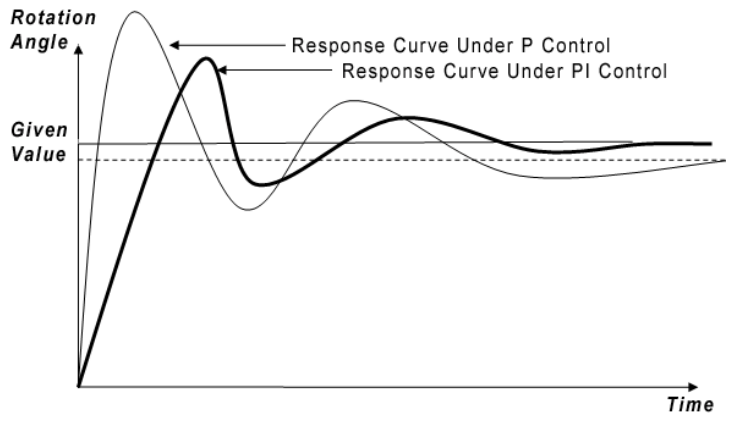

Figure 9. Response of the System under PI Control

\section{The Use of Fuzzy Control to Eliminate the Swing in the Motion of the Servo}

When the sensor detects the position with maximum light intensity, it prefers that the servo could stop at the specified position steadily as soon as possible. On fuzzy control, we set the difference between the current position and the specified location as fuzzy variables (the membership function showed as below), and the rotational speed of servo as controlled variable. Thus when the servo swings, it is able to accelerate first and then decelerate, which improves the stability of the system $[8,9]$.

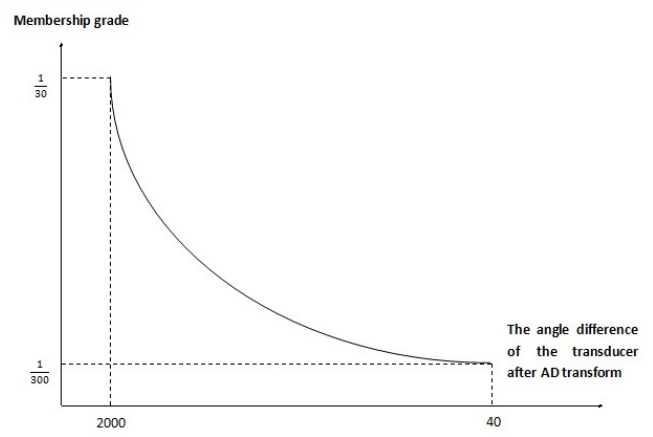

Figure 10. Membership Function Graphic of the Fuzzy Control 


\section{PERFormance And CONClusion}

The test result of the entity structure demonstrates that the daylighting system could meet the basic lighting requirements of the buildings. Nevertheless, during the practice, it is suggested that the system would possibly influenced by the environment factors and the system itself.

\section{A. Environmental Factors}

The position and the structure of buildings, the variance of light intensity at different time and regions would contribute to the lighting effect. Through the simulation measurement, the influence of environmental factors is showed as below. (Light intensity is measured by OPT101 chip, which has an output range from $0 \mathrm{~V}$ to $5 \mathrm{~V}$, and proportionate to the light energy per unit area received.)

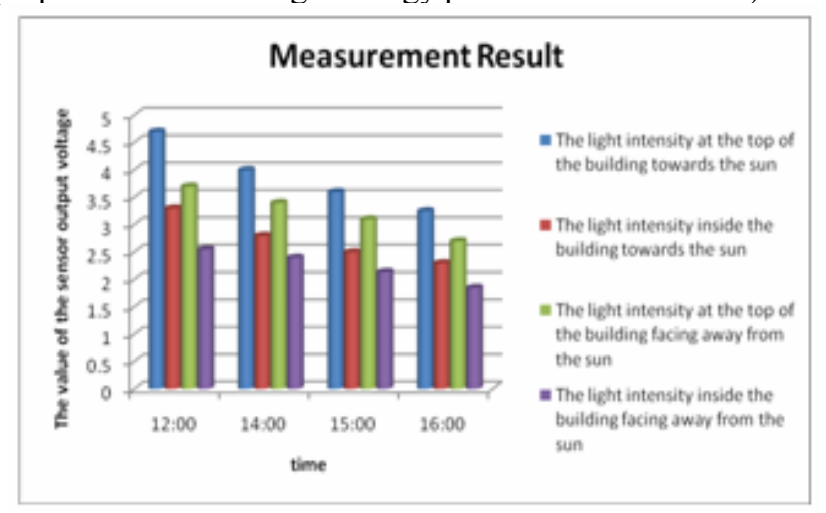

Figure 11. Result of the Simulation Measurement

\section{B. System Factors}

The measurement errors of the sensors could cause the value different from the designed one, which have a great influence on the effect of energy saving as far as the system factors are concerned. The output voltage of sensor voltage comparator circuits that were applied before distorted under the influence of power frequency noise. In particular, when two output voltages were close (The values of the corresponding light intensity were close), the difference signal would change into a power frequency pulse signal with a great jitter. Aimed at improving the performance, the combination of $\mathrm{RC}$ filtering and $\mathrm{A} / \mathrm{D}$ conversion prevents the result from power frequency noise.

\section{REFERENCES}

[1] Chi Zeying, Chen Wenjian. Applied Optics and Elements of Optical Design. Southeast University Press(2008).

[2] Chinese Optics and Applied Optics Abstracts. Changchun Institute of Optics, Fine Mechanics and Physics, Chinese Academy of Sciences (1987 - Present).

[3] Max Born, Emil Wolf. Principles of Optics. Electronic Industry Press (2009).

[4] An Liansheng, Li Lin, Li Quanchen. Applied Optics. Beijing Institute of Technology Press (2008).

[5] Qing Taiquan, Guo Mingqiong. The Selection of Latest Transducers Manual. Chinese Electric Power Press (2009).

[6] Li Changfa. Daylighting and Artificial Lighting of Office Building. China Illuminating Engineering Journal (1996).

[7] Wang Jin, Chen Lin. Power Management System for Intelligent Solar Energy Lighting. Microcontrollers and Embedded Systems (2009).

[8] Wu Chunze, Zhu Sili, Lin Yandan, Sun Yaojie. Fuzzy Control of Natural Light in Lighting. China Illuminating Engineering Journal (2010).

[9] Lang Encai, Chen Yifei. Lighting Fuzzy Controller Based on Visual Comfort Indoors. China Instrumentation (2008). 